\title{
Antibiotic resistance potential of the healthy preterm infant gut microbiome
}

\author{
Graham Rose ${ }^{\text {Corresp.. }}{ }^{1}$, Alexander G Shaw ${ }^{2}$, Kathleen Sim ${ }^{2}$ ， David J Wooldridge ${ }^{1}$ ， Ming-Shi Li ${ }^{2}$, Saheer \\ Gharbia $^{1}$, Raju Misra ${ }^{1}$, John Simon Kroll ${ }^{3}$ \\ ${ }^{1}$ Genomics Research Unit, Public Health England, London, United Kingdom \\ 2 Department of Medicine, Imperial College London, London, United Kingdom \\ 3 Section of Paediatrics, Department of Medicine, Imperial College London, London, United Kingdom \\ Corresponding Author: Graham Rose \\ Email address: graham.rose@phe.gov.uk
}

Background: Few studies have investigated the gut microbiome of infants, fewer still preterm infants. In this study we sought to quantify and interrogate the resistome within a cohort of premature infants using shotgun metagenomic sequencing. We describe the gut microbiomes from preterm but healthy infants, characterising the taxonomic diversity identified and frequency of antibiotic resistance genes detected.

Results: Dominant clinically important species identified within the microbiomes included C. perfringens, $K$. pneumoniae and members of the Staphylococci and Enterobacter genera. Screening at the gene level we identified an average 13 genes per preterm infant, ranging across 8 different antibiotic classes, including aminoglycosides and fluoroquinolones. Some antibiotic resistance genes were associated with clinically relevant bacteria, including the identification of mecA and high levels of Staphylococci within some infants. We were able to demonstrate that in a third of the infants the $S$. aureus identified was unrelated using MLST or metagenome assembly, but low abundance prevented such analysis within the remaining samples.

Conclusions: We found that the healthy preterm infant gut microbiomes in this study harboured a significant diversity of antibiotic resistance genes. This broad picture of resistances and the wider taxonomic diversity identified raises further caution to the use of antibiotics without consideration of the resident microbial communities. 
1 Antibiotic resistance potential of the healthy preterm infant gut microbiome

2 Graham Rose $^{1 \#}$, Alexander G. Shaw ${ }^{2}$, Kathleen $\mathrm{Sim}^{2}$, David J. Wooldridge ${ }^{1}$, Ming-Shi Li ${ }^{2}$, Saheer

3 Gharbia $^{1}$, Raju V. Misra ${ }^{1}$, J. Simon Kroll $^{2}$

$4{ }^{1}$ Genomic Research Unit, Public Health England, Microbiology Services, 61 Colindale Avenue, 5 London, UK

$6{ }^{2}$ Department of Medicine, Section of Paediatrics, Imperial College London, London, UK

$7 \quad{ }^{*}$ Corresponding author. Mailing address:

8 Genomic Research Unit, Public Health England, Microbiology Services, 61 Colindale Avenue, 9 London NW9 5HT, UK.

10 E-mail: graham.rose@phe.gov.uk 


\section{Abstract}

12 Background: Few studies have investigated the gut microbiome of infants, fewer still preterm 13 infants. In this study we sought to quantify and interrogate the resistome within a cohort of 14 premature infants using shotgun metagenomic sequencing. We describe the gut microbiomes

15 from preterm but healthy infants, characterising the taxonomic diversity identified and frequency 16 of antibiotic resistance genes detected.

17 Results: Dominant clinically important species identified within the microbiomes included $C$. 18 perfringens, $K$. pneumoniae and members of the Staphylococci and Enterobacter genera. 19 Screening at the gene level we identified an average 13 genes per preterm infant, ranging across 8 20 different antibiotic classes, including aminoglycosides and fluoroquinolones. Some antibiotic 21 resistance genes were associated with clinically relevant bacteria, including the identification of $22 m e c A$ and high levels of Staphylococci within some infants. We were able to demonstrate that in a 23 third of the infants the S. aureus identified was unrelated using MLST or metagenome assembly, 24 but low abundance prevented such analysis within the remaining samples.

25 Conclusions: We found that the healthy preterm infant gut microbiomes in this study harboured a 26 significant diversity of antibiotic resistance genes. This broad picture of resistances and the wider 27 taxonomic diversity identified raises further caution to the use of antibiotics without 28 consideration of the resident microbial communities.

\section{Introduction}

Over recent years the composition of the gastrointestinal (GI) microbiota has been 31 increasingly implicated in health and disease, with bacterial populations harbouring both 
32 beneficial commensals and pathogens. A diverse bacterial population results in greater genetic 33 content, but some of this additional genetic material is less welcome. Previous studies have 34 implicated the GI microbiota as a reservoir of antimicrobial resistance (AMR) genes (Penders et al., 2013), held by, or capable of being transferred to, potential pathogens. Whilst often benign, during bacterial infection transfer of AMR genes can occur, which - coupled with selection pressures arising through antimicrobial therapy - can make treatment difficult, increasing the time taken to cure the infection. Furthermore, antimicrobial therapies are generally (ideally) tailored towards acute infections targeting a single pathogen, with little consideration of the wider microbial communities which reside in the microbiome, leading to a situation in which the use of antibiotics may cause unintentional harm to the host.

As our understanding of the microbiome has developed, the collection of AMR genes within a bacterial population has recently been defined as the resistome (Penders et al., 2013). Antibiotics have a role in shifting the profile of the resistome within the population (Jernberg et al., 2007), with low antibiotic-use communities harbouring lower AMR gene frequencies (Walson et al., 2001; Bartoloni et al., 2009). Heavy treatment of bacterial populations with antibiotics can lead to the long term overrepresentation of AMR genes. Such dynamics are evident in the microbiome of preterm neonates, who receive multiple antibiotic courses, and are cared for in an

49 Intensive Care Unit environment potentially contaminated with multi-resistant bacteria. Antibiotic treatments for both term and preterm neonates have demonstrated lasting effects on the microbiota (Tanaka et al., 2009; Arboleya et al., 2015), with the trajectory of population development diverging from untreated controls, leading to a potential scenario of prolonged even life-long - high frequency AMR reservoirs through the selection of bacteria within the population that are most resistant. A wide range of AMR genes have been found in neonatal

55 populations (de Vries et al., 2011; Zhang et al., 2011), some shown to be present from birth 
56 (Alicea-Serrano et al., 2013; Gosalbes et al., 2015), whilst twin pairs have been shown to have GI

57 communities with similar distributions of both organisms and resistance genes (Moore et al.,

58 2015). These observations suggest vertical transmission as a source, with discrepancies between

59 mothers and babies being due to the substantial shifts in the microbiota adapting to the very

60 different environment of a newly born infant's GI tract (Gosalbes et al., 2015).

61 The GI tract of a premature neonate is a particularly unusual scenario for observation of

62 AMR genes, due to greatly reduced bacterial immigration as a result of the isolated, sterile

63 environment of incubators and very controlled enteral feeds; donor breast milk may be

64 pasteurised and, whilst unpasteurised maternal milk (which harbours specific bacteria (Beasley \&

65 Saris, 2004; Jimenez et al., 2008; Martin et al., 2009)) is given where possible, there is a

66 likelihood of little or no breastfeeding due to extreme prematurity.

67 In these circumstances, the GI community and the resistance genes present are likely in

68 the main to be derived from the mother, and acquired during birth. Whilst limited bacterial 69 numbers and diversity will initially be transferred, mechanisms are available for the 70 dissemination of AMR through the expanding bacterial population (as reviewed by van Hoek et 71 al (2011)) with transfer having been documented within the gut environment (Shoemaker et al.,

72 2001; Karami et al., 2007; Trobos et al., 2009). Heavy use of antibiotics in the course of care of

73 premature infants would not only then skew the bacterial population and drive resistance 74 selection, but has been shown to increase the activity of some transposable elements due to 75 stressing of bacterial populations (Beaber, Hochhut \& Waldor, 2004).

In this study, we present a detailed investigation of the resistome from the GI microbiota 77 of eleven premature infants, with detailed information on antibiotic receipt and maternal 78 antibiotic use. The microbiota of premature infants has been subjected to such investigations 79 before, but through targeted techniques such as PCR or qPCR (Gueimonde, Salminen \& Isolauri, 
80 2006; Alicea-Serrano et al., 2013; von Wintersdorff et al., 2016) or through functional

81 metagenomics (de Vries et al., 2011; Moore et al., 2015), which has the disadvantage of not being

82 able to quantify the antibiotic resistance potential of a community (Forslund et al., 2014). We

83 have used shotgun metagenomic sequencing to describe the resistome in its entirety, moving from

84 species level taxonomic profiling, to characterisation of the resistance landscape, including typing

85 of metagenomes identified as potentially harbouring $m e c A$, conferring resistance to methicillin

86 and other $\beta$-lactam antibiotics.

\section{Materials \& Methods}

\section{Study population}

The study was approved by West London Research Ethics Committee (REC) Two, United

90 Kingdom, under the REC approval reference number 10/H0711/39. Parents gave written

91 informed consent for their infant to participate in the study.

Faecal samples analysed were collected from premature infants, defined as less than 32 completed weeks of gestation. Premature infants were recruited to the study at the Imperial

94 College Healthcare National Health Service Trust neonatal intensive care unit (NICU), at Queen

95 Charlotte's and Chelsea Hospital, between January 2010 and December 2011.

\section{Sample collection}

97 Almost every faecal sample produced by each participant between recruitment and

98 discharge was collected by nursing staff from diapers using a sterile spatula. Samples were placed

99 in a sterile DNase-, RNase-free Eppendorf tube, stored at $-20^{\circ} \mathrm{C}$ within two hours of collection 
100 and stored at $-80{ }^{\circ} \mathrm{C}$ within five days. A single faecal sample from each of twelve infants who had

101 no diagnosis of necrotising enterocolitis or blood-stream infection during their admission was

102 selected for metagenomic sequencing. DNA from one faecal sample did not complete library

103 preparation (see below); clinical characteristics of the remaining eleven infants and faecal sample

104 metadata are presented in Table S1.

105 DNA extraction and shotgun library preparation

106 DNA extractions were performed as described previously (Rose et al., 2015), but with the 107 following modifications: DNA extracts were prepared from approximately $200 \mathrm{mg}$ of faeces, 108 which were re-suspended in 10x volume:weight filtered 1x phosphate-buffered saline (PBS), with 109 addition of 1:1 (volume:volume) 2\% 2-mercaptoethanol diluted in 1x filtered PBS. The MolYsis 110 selective lysis kit (Molzym) was used for the selective lysis of eukaryotic cells, incorporating the 111 modifications previously described (Rose et al., 2015). Bacterial lysis was performed by addition 112 of $50 \mu 1$ lysozyme (Sigma), $6 \mu 1$ mutanolysin (Sigma) and $3 \mu 1$ lysostaphin (Sigma) to $100 \mu 1$ of 113 re-suspended bacterial pellet, and incubated at $37^{\circ} \mathrm{C}$ for $1 \mathrm{~h}$. This was followed by addition of 2 $114 \mu \mathrm{l}$ proteinase $\mathrm{K}$ and $150 \mu 12 \mathrm{x}$ Tissue and Cell lysis buffer (Epicentre) and incubated at $65^{\circ} \mathrm{C}$ for $11530 \mathrm{~min}$. Lysates were added to $2 \mathrm{ml}$ tubes containing $0.25 \mathrm{ml}$ of $0.5 \mathrm{~mm}$ beads and beaten on a 116 Fast Prep 24 system at $6 \mathrm{~m} / \mathrm{s}$ for $20 \mathrm{~s}$ and repeated once after $5 \mathrm{~min}$. Finally, DNA was purified 117 using the MasterPure complete kit (Epicentre) according to the manufacturer's instructions, 118 eluted in $50 \mathrm{ul} 0.1 \times$ TE buffer (Sigma) and stored at $-80{ }^{\circ} \mathrm{C}$.

119 Extracted DNA was fragmented using the NEBNext dsDNA fragmentase kit (NEB) according to the manufacturer's instructions. Shotgun DNA libraries were subsequently prepared 121 using the KAPA HyperPrep kit (KAPA Biosystems) according to the manufacturer's instructions. 
122 Ligated libraries were amplified by PCR with the number of cycles being dependant on starting 123 material biomass, varying between 2 and 8 (mean 3 cycles). A negative extraction control was 124 included consisting of $1 \mathrm{ml}$ filtered 1x PBS and processed alongside the samples. After library 125 amplification, the negative extraction control and one preterm infant faecal sample required $>8$ 126 PCR cycles owing to very low starting pre-PCR biomass (DNA concentration $<0.1 \mathrm{ng} / \mathrm{ul}$ ), 127 therefore these samples were excluded from downstream analysis, leaving faecal samples from 128 eleven premature infants.

\section{Shotgun metagenomic sequencing}

Library insert size and quantity was assessed for each sample by Bioanalyser and qPCR as described previously (Rose et al., 2015). Library insert size ranged from 244 bp to 288 bp with a mean of $261 \mathrm{bp}$. Libraries were sequenced on either an Illumina NextSeq 500 system or part of replicate runs on an Illumina MiSeq system. Prior to loading, libraries were normalised, pooled and diluted to either $1.6 \mathrm{pM}$ or $18 \mathrm{pM}$ for sequencing on the NextSeq or on the MiSeq, respectively. Paired end (PE) sequencing was performed on the NextSeq using a v2 300 cycle high output reagent kit (Illumina) and on the MiSeq using the v3 600 cycle reagent kit (Illumina). were sequenced as part of separate multiplexed MiSeq runs, generating a mean 8.3 million PE reads and 5.0 Gbp sequence yield per sample. Later sequencing on a NextSeq instrument 140 included the complete 11 sample set, and inclusion of a technical replicate (sample Q89). A mean 14110.0 million PE reads were generated per sample, yielding 3.0 Gbp. 


\section{Sequencing data availability}

143 All sequencing data generated for this study is available from the EBI European 144 Nucleotide Archive, under study accession $\quad$ PRJEB15257 (see 145 http://www.ebi.ac.uk/ena/data/view/PRJEB15257).

\section{Processing of metagenomic sequences}

Sequence quality was calculated using FastQC (v0.11.3) (Andrews, 2010). Read filtering

148 was performed using Trimmomatic (v0.32) (Bolger, Lohse \& Usadel, 2014). This consisted of 149 adapter sequence removal based on an in house database of Illumina adapters, primers and index 150 sequences using non-default parameters (ILLUMINACLIP 2:30:10), and subsequent read 151 trimming consisting of an initial head crop of the first $15 \mathrm{bp}$, then iterative removal of leading 152 and trailing bp with phred qualities $<20$, and internal bases where mean base phred qualities $<20$ 153 in 4 bp sliding windows (parameters were: LEADING:20 TRAILING:20 154 SLIDINGWINDOW:4:20). Finally sequences with less than 40 bp remaining were discarded 155 (MINLEN:40).

A mapping based approach was used to remove expected human host sequences from the 157 faecal samples, as well as any remaining vector contamination. Using FastQ Screen (v0.4.4) 158 (Babraham Institute) and the short read aligner Bowtie2 (v2.2.6) (Langmead \& Salzberg, 2012), 159 reads were mapped against the human genome (GRCh38) and the UniVec (version 8) vector 160 database (non-default parameters were: --aligner bowtie2, --nohits). All unmapped PE reads were 161 output as new fastq files and continued within downstream analysis. 
164 (Magoč \& Salzberg, 2011) (--max-overlap 200), thus generating longer single sequences (mean 165 length $207 \mathrm{bp}$ ).

\section{Species identification and relative abundances}

Primary metagenomic profiling was performed using DIAMOND (v.0.7.9.8) (Buchfink, 168 Xie \& Huson, 2014) and MEGAN (v5.10.6) (Huson et al., 2007). All forward reads, or joined 169 reads in the case of the MiSeq dataset, were aligned against a protein reference database under 170 default parameters. The protein database was built using all 73,055,898 sequences from the NCBI 171 non-redundant (nr) database (downloaded 21-10-15).

173 was performed within MEGAN and under non-default parameters. Sequences were assigned to 174 the NCBI Taxonomy (1,266,115 individual taxa) using the Lowest Common Ancestor (LCA) and 175 the following thresholds: minimum bit-score: 80; $\max$ expectation value: $1.0 \times 10^{-6}$; top 176 percentage of hits considered: 10\%; minimum taxon support based on all assigned reads: $0.01 \%$.

177 Relative abundances and extraction of species specific binned reads were calculated within 178 MEGAN.

A secondary taxonomic profiling method, MetaPhlAn (v.2.2.0) (Truong et al., 2015) was used on all samples with the following parameters: --mpa_pkl metaphlan2/db_v20/mpa_v20_m200.pkl --bowtie2db metaphlan2/db_v20/mpa_v20_m200

182 --input_type fastq. Relative abundance tables were combined using the packaged MetaPhlAn 183 script - merge_metaphlan_tables.py. 


\section{Identifying antimicrobial resistance genes}

185

186

Presence/absence testing of AMR genes within the samples was performed on unassembled reads. Reads were mapped using bowtie2 (v.2.2.6) (Langmead \& Salzberg, 2012) as part of SRST2 (v0.1.7) (Inouye et al., 2014) under default parameters to a clustered ARG-Annot database of acquired resistance genes (Gupta et al., 2014) and all hits recorded (--gene_db srst2/data/ARGannot.rl.fasta). Default parameters set AMR gene reporting at 90\% minimum coverage cutoff. Computational $S$. aureus sequence typing (ST) was also performed using SRST2 (Inouye et al., 2014) under default scoring parameters, and using the $S$. aureus MLST schema downloaded on 18-04-16 from pubmlst.org. Alleles were flagged uncertain when below threshold depths (--min_edge_depth 2, --min_depth 5).

\section{Metagenome assembly and $S$. aureus phylogeny}

Assemblies were performed using spades (v3.7.1) under default parameters except identification of the data as metagenomic (--meta). Assembled contigs were used as blastn queries against the NBCI nt database, and taxonomic labels attached using MEGAN, with all contigs identified as S. aureus (NCBI taxon id: 1280) including summarised contigs extracted per sample. As a reference, all S. aureus complete genomes were downloaded from PATRIC (Release May 2016), totalling 118 genomes.

An anchor based phylogenetic method, andi (v.0.10) (Haubold, Klötzl \& Pfaffelhuber, 2015), was used to estimate the evolutionary distances between the study and global S. aureus genomes set, using Phylip (v.3.696) (Felsenstein, 2009) to infer the neighbour-joining phylogeny. Following assembly, very short binned $S$. aureus contigs $(<1 \mathrm{~kb})$ and partial assemblies, in this case those with less than half of the median $S$. aureus genome size $(<1.5 \mathrm{Mb})$, were excluded 
206 from andi and phylogenetic tree construction as based on recommended guidelines (Haubold, 207 Klötzl \& Pfaffelhuber, 2015).

\section{S. aureus typing}

Experimental confirmation of $m e c A$ was attempted for all eleven samples. Faecal samples were cultivated on the Staphylococcal selective growth media manitol salt agar. Sweeps of the presumptive Staphylococcus colonies from each sample were propagated and extracted by the 212 following protocol: half a $10 \mu \mathrm{l}$ loop of overnight growth at $35^{\circ} \mathrm{C}$ was inoculated into $2 \mathrm{ml}$ tubes containing $0.5 \mathrm{~mm}$ silica/ zirconia beads filled to the $0.25 \mathrm{ml}$ mark and $350 \mu \mathrm{l}$ of Master Pure Tissue Cell Lysis Buffer (EpiCentre). Bead beating was performed using a Fast Prep (MPBio) at $6 \mathrm{~m} / \mathrm{s}$ for 20 seconds. This was repeated three times with a 5 minute pause between each pulse. Lysates were centrifuged at $8,000 \mathrm{xg}$ for 10 minutes and $300 \mu \mathrm{l}$ of supernatant transferred to a new tube. A known mecA positive strain (NCTC strain 12232) and a mecA negative clinical isolate were used as control strains.

A multiplexed PCR method was used to type the SCCmec element within the samples according to the protocol described previously (Milheiriço, Oliveira \& De Lencastre, 2007), but with the following exceptions. Each $50 \mu \mathrm{l}$ PCR reaction consisted of 1x HotStart Ready Mix (KAPA Biosystems), $25 \mathrm{ng}$ genomic DNA, and primers at the described concentration. The cycling conditions were as follows: $95{ }^{\circ} \mathrm{C}$ for 3 minutes followed by 30 cycles of $98{ }^{\circ} \mathrm{C}$ for 20 seconds, $53{ }^{\circ} \mathrm{C}$ for 30 seconds and $72{ }^{\circ} \mathrm{C}$ for 30 seconds followed by a final extension of $72{ }^{\circ} \mathrm{C}$ for 4 minutes. Amplicons were purified using the AgenCourt AMpure XP PCR purification kit (Beckman Coulter) following manufacturer's instructions. Amplicon sizes were determined measured by BioAnalyser (Agilent) on a high sensitivity DNA chip, with classification of a 
228

229 230

positive result based on on fragment sizes \pm 5 bp of those expected, and peak concentration $>=$ $500 \mathrm{pg} / \mathrm{ul}$. In addition to the above controls, extraction and PCR negative controls were included, which substituted input genomic DNA for purified water.

\section{Statistics}

Species richness and the evenness of their abundance were quantified using the ShannonWeaver index ecological measure, calculated within MEGAN. Visualisation of samples was performed by hierarchical clustering using the UPGMA method and principal coordinates analysis (PCoA), all based on a matrix of Bray-Curtis distances calculated within MEGAN. Correlations and t-tests were performed within R (v 3.2.5) (R Developement Core Team, 2015).

\section{Results}

\section{The healthy preterm metagenome}

Using shotgun metagenomic sequencing we have captured an early snapshot of the antimicrobial resistance landscape within the gut microbiota of eleven premature infants who did not have proven sepsis or necrotizing enterocolitis. Infants were born either vaginally $(\mathrm{N}=6)$ or by caesarean section $(\mathrm{N}=5)$, with gestational ages ranging 24-31 weeks (mean 26.9 weeks). Ages of the infants at which the samples were taken ranged from 5 - 43 days (mean 25.7 days) (Table S1). A mixture of benchtop to medium throughput Illumina platforms were used to generate a dataset of 145.6 million paired end (PE) reads (51.4 Gbp sequence data) (Table 1), enabling us to characterise taxonomic and antimicrobial resistance profiles. 

analysis with filtering by the Lowest Common Ancestor (Huson et al., 2007; Buchfink, Xie \& 249 Huson, 2014), which enabled assignment of taxonomic labels for $71.5 \%$ of the reads within the complete dataset to at least the level of Kingdom (Table S2). As an alternative method, we also 251 profiled the dataset using a marker based approach (Truong et al., 2015), which was highly congruent to species level relative abundances, as well as higher taxonomic ranks, to the blast

253 based method used (Pearson $\mathrm{R}=0.9$ - 1.0) (Table S3). Replicate sequencing of samples also 254 demonstrated reproducibility of the method, either by cluster analysis (Figure 1) or pairwise 255 correlations (Figure S1). species, such as sample Q216 with 85.1\% Clostridium perfringens, Q189 with $73.1 \%$ Klebsiella pneumoniae, and Q83 with 85.9\% Enterococcus faecalis (Figure 1A). In terms of prevalence, the previous three species, as well as Enterobacter cloacae and Staphylococcus epidermidis, were found at over $50 \%$ relative abundance in one or more samples. Furthermore, S. epidermidis and

S. aureus were ubiquitous, ranging from $0.06 \%$ to $57.1 \%$ abundance in all samples (Figure 1B). Principal coordinate analysis (PCoA) demonstrated three loose sample groups based on a high abundance of S. epidermidis, K. pneumoniae, and either B. breve, S. aureus or C. perfringens

264 (Figure 1C). In total we identified a non-redundant set of 172 species across all samples (see 265 Table S4 for complete dataset).

\section{Prevalence of antimicrobial resistance}


269 antibiotic exposure of the preterm infants (Figure S2). Eight of the eleven infants had received a

270 course of prophylactic antibiotic treatment consisting of co-amoxiclav (Table S1), whilst a second

271 course was administered to four infants, consisting of combinations of co-amoxiclav, tazocin or

272 vancomycin. In total, exposure ranged from 2 - 8 days of antibiotics before samples were taken,

273 excluding infants Q87 and Q89 which received no antibiotics. Antibiotics were also administered

274 maternally to three infants (Q26, Q117 \& Q189), but this did not include the two above infants

275 with no antibiotic treatment. Sample diversity ranged from $0.9-2.9(\mathrm{SD} \pm 0.5)$, but when

276 compared to cumulative antibiotic exposure expressed in days, no significant difference was

277 found between the taxonomic diversity and amount of antibiotic exposure for untreated and 278 treated infants (unpaired $t$ test, $P=0.17$ ) (Figure S2). It is important to stress however that the

279 small and heterogeneous nature of the sample set will have reduced the power to detect 280 differences between antibiotic exposure in this study, and so prevented any meaningful 281 stratification by other clinical variables such as mode of delivery or day of life.

A mapping based approach against a comprehensive collection of acquired antibiotic resistance genes was next used to quantify AMR within the eleven metagenomes (Inouye et al., 2014). In total 143 AMR genes were identified, consisting of a non-redundant set of 39 different AMR genes (Figure 2 and Table S5). Per infant, the average number of genes identified was 13 (ranging 5 - 22 genes), and AMR genes were found across eight different antibiotic classes, sequence database was $99.0 \%$, and sequence divergence ranged from no difference to $12.3 \%$

289 (Table S6). In total over 1,600 alleles were searched for, and notable AMR genes not detected 290 included those involved in carbapenem and vancomycin resistance, the latter of which was 291 administered to three preterm infants prior to sample collection (Table S1). The class most

292 frequently detected were $\beta$-lactamases, comprising ten different genes (Table 2), of which the 
293 blaZ gene was present in every infant. Interestingly, within this set of $\beta$-lactamasae genes, mec $A$

294 was found in four infants (Q87, Q117, Q175, and Q189), and at a mean depth of coverage 295 ranging from 3.9 to $52.2 \mathrm{bp}$ (Figure 2). mec $A$ confers resistance to methicillin as well as other $\beta$ -

296 lactam antibiotics, and is carried on the SCCmec mobile element found across several

297 Staphylococci species. Identification of four infants with potential methicillin resistant S. aureus

298 (MRSA) or S. epidermidis (MRSE) carriage, along with high abundances and prevalence of both

299 S. aureus and S. epidermidis species across the dataset, could indicate a significant reservoir for 300 AMR transfer between the species, as well as highlight the seeding of the infant gut microbiome 301 from an early stage.

\section{Focus on $S$. aureus species detected}

We next wanted to understand the relationship of the $S$. aureus species within the mecA positive as well as negative samples, as the premature infants overlapped in time and so could harbour closely related strains. This was undertaken to firstly confirm in silico prediction of mec $A$ using an established molecular based typing method, but also to push the metagenomic analysis further on what was known to be a challenging dataset owing to the range of identified S. aureus as described above, with relative abundance ranging from $0.06 \%-39.8 \%$ (Table S4).

We first tested the computational prediction of mecA experimentally using a multiplexed PCR typing method (Milheiriço, Oliveira \& De Lencastre, 2007), which provides detection of the $m e c A$ gene, in addition to typing of the mobile element carrying the gene ( $\mathrm{SCCmec}$ ), although this component of the assay was beyond the scope of this study. Using this method we detected mecA presence correctly within the control strains, a methicillin resistant (MRSA) and susceptible (MSSA) strain (see Methods), and three out of the four predicted mecA positive samples generated a positive mecA result (Table S7). The exception, sample Q87, generated the 
316 expected mecA amplicon size but the concentration of this fell below the threshold for detection $317(<500 \mathrm{pg} / \mathrm{ul})$ and so was excluded.

In an attempt to understand strain relatedness directly from the metagenomic data we undertook in silico MLST analysis using an S.aureus schema as well as metagenome assembly.

320 The MLST was able to classify four of the eleven samples, all with different ST types - ST8, 321 ST1027, ST22, ST25, although the last two had some degree of uncertainty in their assignment 322 (Table S8). This suggests that for at least these four samples, the S. aureus strains are unrelated 323 and unlikely a result of transmission. We were interested to know if de novo assembly of the 324 metagenome could be utilised to resolve these and any of the remaining unclassifiable samples 325 further. Following assembly and identification of $S$. aureus contigs (see Methods), we found that 326 it was not possible to capture more than a fifth of the expected genome size for the above 327 unclassified samples, with an abundance of $>3 \%$ necessary to achieve over $90 \%$ estimated 328 capture, which was achieved in four cases (Table S9). Phylogenetic reconstruction of these four 329 genomes alongside a collection of published $S$. aureus genomes (Table S10), provided 330 confirmation of the diversity of $S$. aureus identified (Figure S3), enabling placement across a 331 global collection of strains.

\section{Discussion}

It is recognised that one of the most important public health threats worldwide is antimicrobial resistance. Here we report on the gut composition and AMR diversity for eleven healthy but premature infants. Recent studies have shown that the initial seeding of the infant gut microbiome is influenced by the microorganisms in the immediate environment, and whilst

337 colonisation by bacteria with AMR genes has been demonstrated (Brooks et al., 2014), 
338 comparatively far fewer studies have investigated the gut microbiome of infants, fewer still

339 preterm healthy infants. Interest has also increased on how the trajectory of the early gut 340 microbiome is influenced to form the 'stable' adult microbiome. The preterm infant gut 341 microbiome is very different compared to full-term infants (Groer et al., 2014), displaying a 342 much lower diversity, particularly in anaerobes, with an increase in coagulase-negative 343 Staphylococci and Enterobacteriaceae (Adlerberth \& Wold, 2009); adult microbiomes are 344 characterised by several hundred, mostly anaerobic bacterial species (Adlerberth \& Wold, 2009). 345 We found a similarly low level of species diversity across all metagenomes, with each sample 346 dominated by a few highly abundant species, including $C$. perfringens, $K$. pneumoniae and 347 members of the Staphylococci and Enterobacter genera. Presence of such species are in common 348 with previous studies on the premature gut microbiome (Groer et al., 2014; Gibson et al., 2016).

Interestingly, each metagenome profile displayed a different dominant few species, 350 clustering into three loose groupings. This could reflect the dynamic nature of the early 351 establishing gut, with the preterm infant microbiome acquiring an increased diversity of bacteria 352 and subjected to a great amount of change until it matures into what is recognised as a more 353 'stable' microbiome. Although we found no correlation between diversity and antibiotic 354 exposure, with infants treated with either no antibiotics (including during pregnancy), to up to 8 355 days of antibiotic administration, effects such as relatively small sample size, as well as day of 356 life of sample and normal gut development are biases to this finding, which is contrary to other 357 studies within infants (Greenwood et al., 2014; Merker et al., 2015). It could be that at this very 358 early stage, the microbiota is influenced to a greater extent by seeding during birth from the 359 mother and environment than antibiotic treatment, or that not enough time has passed to detect 360 differences from the antibiotics administered; larger sample numbers would be required, 
361 alongside longitudinal studies and parallel maternal sampling to better understand the 362 development of diversity.

A threat to this development is the acquisition of antibiotic resistant bacteria, which can 364 potentially seed the infant microbiome. Coupled with the high rate of horizontal gene transfer 365 within the commensal community (Stecher et al., 2012), the preterm infant gut microbiome has 366 the potential to be a reservoir for AMR. With dominance of the preterm gut by species known to 367 carry clinically relevant antibiotic resistance, we next quantified the burden of antibiotic 368 resistance genes within the infant's faecal flora, which identified an average 13 genes per infant. 369 Previous targeted or functional studies based on infants have found some of the AMR genes also 370 identified here, including those for Tetracycline (tet) (Gueimonde, Salminen \& Isolauri, 2006; 371 Alicea-Serrano et al., 2013) and $\beta$-lactam (bla) (Fouhy et al., 2014). In a wider context, it is 372 known that AMR genes are a common feature of bacterial populations, found in communities 373 inhabiting the soil, rivers and even deep-sea sediment (Knapp et al., 2010; Qin et al., 2011; 374 Kittinger et al., 2016). Therefore, whilst their presence in the human gut microbiome should be of 375 little surprise (Bailey et al., 2010), identification of genes such as mecA demonstrates the prevalence of some clinically significant resistant bacteria from birth. generated, enabling multiple avenues of questions to be addressed. However, short read sequencing remains a challenge when applied to the linkage of resistance elements, such $m e c A$, to specific genome sequences (strains), which is made difficult by the nature of metagenomic samples containing multiple alleles from different closely related species, as well as potentially multiple strains of the same species. Secondly, the methods used here were inherently restricted to identification of known AMR genes found within the ARGannot database used in this study, 384 which contains those genes involved in acquired resistance only, therefore chromosomal 
385 mutations, such as those conferring resistance to rifampicin as well as novel resistance genes

386 would have been missed, leading to potential underrepresentation of resistance in this study.

\section{Conclusions}

388 The healthy preterm infants sampled within this study harboured multiple AMR genes, 389 representing a potential reservoir for later disease onset. In particular, detection of clinically 390 important AMR genes, such as mecA, highlights the need to further understand the impact that 391 this reservoir could have on later treatment regimes.. From a methodology point, this approach 392 was able to provide a comprehensive snapshot of the complete taxonomic diversity and resistome 393 in one assay. Although tracking of the movement of such AMR genetic elements would be 394 enhanced by improved handling of the dynamic ranges of abundances; different methods at the 395 level of sample preparation, such as sample normalisation, may offer potential answers to such hurdles. Overall this study leads to questions such as how this resistance potential contributes to

397 later clinical intervention or disease onset, and if antibiotic treatment without knowledge of prior 398 AMR burden could lead to unintentional harm. More broadly, this and other studies show the 399 great promise that shotgun metagenomics holds for clinical microbiology.

\section{Additional Information}

\section{Abbreviations}

402 AMR, antimicrobial resistance; GI, gastrointestinal; PCR, polymerase chain reaction; qPCR, 403 quantitative polymerase chain reaction; BLAST, basic local alignment search tool; SCCmec, 404 staphylococcal chromosome cassette mec; LCA, lowest common ancestor; MLST, multilocus 
405 sequence typing; MALDI-TOF, Matrix-assisted laser desorption/ionization - time of flight; 406 PCoA, principal coordinates analysis.

\section{References}

408 Adlerberth I., Wold AE. 2009. Establishment of the gut microbiota in Western infants. Acta

409 Paediatrica, International Journal of Paediatrics 98:229-238. DOI: 10.1111/j.1651-

$410 \quad 2227.2008 .01060 . x$.

411 Alicea-Serrano AM., Contreras M., Magris M., Hidalgo G., Dominguez-Bello MG. 2013.

412 Tetracycline resistance genes acquired at birth. Archives of Microbiology 195:447-451. DOI:

$413 \quad 10.1007 / \mathrm{s} 00203-012-0864-4$.

414 Andrews S. 2010.FastQC: A quality control tool for high throughput sequence data.

415 Arboleya S., Sanchez B., Milani C., Duranti S., Solis G., Fernandez N., De Los Reyes-Gavilan

416 CG., Ventura M., Margolles A., Gueimonde M. 2015. Intestinal microbiota development in

417 preterm neonates and effect of perinatal antibiotics. Journal of Pediatrics 166:538-544.

$418 \quad$ DOI: $10.1016 /$ j.jpeds.2014.09.041.

419 Bailey JK., Pinyon JL., Anantham S., Hall RM. 2010. Commensal Escherichia coli of healthy 420 humans: A reservoir for antibiotic-resistance determinants. Journal of Medical Microbiology 59:1331-1339. DOI: 10.1099/jmm.0.022475-0.

422 Bartoloni A., Pallecchi L., Rodríguez H., Fernandez C., Mantella A., Bartalesi F., Strohmeyer M.,

423 Kristiansson C., Gotuzzo E., Paradisi F., Rossolini GM. 2009. Antibiotic resistance in a very

424 remote Amazonas community. International Journal of Antimicrobial Agents 33:125-129.

425 DOI: 10.1016/j.ijantimicag.2008.07.029. 
426 Beaber JW., Hochhut B., Waldor MK. 2004. SOS response promotes horizontal dissemination of 427 antibiotic resistance genes. Nature 427:72-74. DOI: 10.1038/nature02241.

428 Beasley SS., Saris PEJ. 2004. Nisin-Producing Lactococcus lactis Strains Isolated from Human 429 Milk. Applied and environmental microbiology 70:5051-5053. DOI:

430

Bolger AM., Lohse M., Usadel B. 2014. Trimmomatic: A flexible trimmer for Illumina sequence data. Bioinformatics 30:2114-2120. DOI: 10.1093/bioinformatics/btu170.

Brooks B., Firek BA., Miller CS., Sharon I., Thomas BC., Baker R., Morowitz MJ., Banfield JF. 2014. Microbes in the neonatal intensive care unit resemble those found in the gut of

Buchfink B., Xie C., Huson DH. 2014. Fast and sensitive protein alignment using DIAMOND. premature infants. Microbiome 2:1. DOI: 10.1186/2049-2618-2-1. Nature methods 12:59-60. DOI: 10.1038/nmeth.3176.

Felsenstein J. 2009. PHYLIP - Phylogeny Inference Package, Version 3.69. (Seattle, WA: University of Washington). The American Naturalist 171:713-725. DOI: 10.1111/j.10960031.1989.tb00562.x.

Forslund K., Sunagawa S., Coelho LP., Bork P. 2014. Metagenomic insights into the human gut resistome and the forces that shape it. BioEssays 36:316-329. DOI: 10.1002/bies.201300143.

Fouhy F., Ogilvie LA., Jones B V., Ross RP., Ryan AC., Dempsey EM., Fitzgerald GF., Stanton C., Cotter PD. 2014. Identification of aminoglycoside and $\beta$-lactam resistance genes from within an infant gut functional metagenomic library. PLoS ONE 9. DOI: 
448 Gibson MK., Wang B., Ahmadi S., Burnham C-AD., Tarr PI., Warner BB., Dantas G. 2016.

449 Developmental dynamics of the preterm infant gut microbiota and antibiotic resistome.

450 Nature Microbiology:16024. DOI: 10.1038/nmicrobiol.2016.24.

451 Gosalbes MJ., Vallès Y., Jiménez-Hernández N., Balle C., Riva P., Miravet-Verde S., de Vries

452 LE., Llop S., Agersø Y., Sørensen SJ., Ballester F., Francino MP. 2015. High frequencies of

453 antibiotic resistance genes in infants' meconium and early fecal samples. Journal of

454 Developmental Origins of Health and Disease:1-10. DOI: 10.1017/S2040174415001506.

455 Greenwood C., Morrow AL., Lagomarcino AJ., Altaye M., Taft DH., Yu Z., Newburg DS., Ward

456 D V., Schibler KR. 2014. Early empiric antibiotic use in preterm infants is associated with

457 lower bacterial diversity and higher relative abundance of enterobacter. Journal of

458 Pediatrics 165:23-29. DOI: 10.1016/j.jpeds.2014.01.010.

459 Groer MW., Luciano AA., Dishaw LJ., Ashmeade TL., Miller E., Gilbert JA. 2014. Development

460 of the preterm infant gut microbiome: a research priority. Microbiome 2:38. DOI:

461 $10.1186 / 2049-2618-2-38$.

462 Gueimonde M., Salminen S., Isolauri E. 2006. Presence of specific antibiotic (tet) resistance 463 genes in infant faecal microbiota. FEMS Immunology and Medical Microbiology 48:21-25.

464 DOI: $10.1111 /$ j.1574-695X.2006.00112.x.

465 Gupta SK., Padmanabhan BR., Diene SM., Lopez-Rojas R., Kempf M., Landraud L., Rolain J-M.

466 2014. ARG-ANNOT, a new bioinformatic tool to discover antibiotic resistance genes in

467 bacterial genomes. Antimicrobial agents and chemotherapy 58:212-20. DOI:

$468 \quad$ 10.1128/AAC.01310-13.

469 Haubold B., Klötzl F., Pfaffelhuber P. 2015. Andi: Fast and accurate estimation of evolutionary 
470 distances between closely related genomes. Bioinformatics 31:1169-1175. DOI:

$471 \quad$ 10.1093/bioinformatics/btu815.

472 van Hoek AHAM., Mevius D., Guerra B., Mullany P., Roberts AP., Aarts HJM. 2011. Acquired

473 antibiotic resistance genes: An overview. Frontiers in Microbiology 2:203. DOI:

$474 \quad 10.3389 /$ fmicb.2011.00203.

475 Huson DH., Auch AF., Qi J., Schuster SC. 2007. MEGAN analysis of metagenomic data.

$476 \quad$ Genome Research 17:377-386. DOI: 10.1101/gr.5969107.

477 Inouye M., Dashnow H., Raven L-A., Schultz MB., Pope BJ., Tomita T., Zobel J., Holt KE. 2014.

478 SRST2: Rapid genomic surveillance for public health and hospital microbiology labs.

479 Genome medicine 6:90. DOI: 10.1186/s13073-014-0090-6.

480 Jernberg C., Lofmark S., Edlund C., Jansson JK. 2007. Long-term ecological impacts of 481 antibiotic administration on the human intestinal microbiota. Isme.J. 1:56-66. DOI:

$482 \quad$ 10.1038/ismej.2007.3.

483 Jimenez E., Delgado S., Fernandez L., Garcia N., Albujar M., Gomez A., Rodriguez JM. 2008.

484 Assessment of the bacterial diversity of human colostrum and screening of staphylococcal

485 and enterococcal populations for potential virulence factors. Research in Microbiology

486 159:595-601. DOI: 10.1016/j.resmic.2008.09.001.

487 Karami N., Martner A., Enne VI., Swerkersson S., Adlerberth I., Wold AE. 2007. Transfer of an 488 ampicillin resistance gene between two Escherichia coli strains in the bowel microbiota of 489 an infant treated with antibiotics. Journal of Antimicrobial Chemotherapy 60:1142-1145.

$490 \quad$ DOI: $10.1093 / \mathrm{jac} / \mathrm{dkm} 327$.

491 Kittinger C., Lipp M., Baumert R., Folli B., Koraimann G., Toplitsch D., Liebmann A., Grisold 
Knapp CW., Dolfing J., Ehlert PAI., Graham DW. 2010. Evidence of increasing antibiotic resistance gene abundances in archived soils since 1940. Environmental Science and Technology 44:580-587. DOI: 10.1021/es901221x.

Langmead B., Salzberg SL. 2012. Fast gapped-read alignment with Bowtie 2. Nature Methods

Magoč T., Salzberg SL. 2011. FLASH: fast length adjustment of short reads to improve genome assemblies. Bioinformatics (Oxford, England) 27:2957-63. DOI:

Martin R., Jimenez E., Heilig H., Fernandez L., Marin ML., Zoetendal EG., Rodriguez JM. 2009. Isolation of bifidobacteria from breast milk and assessment of the bifidobacterial population by PCR-denaturing gradient gel electrophoresis and quantitative real-time PCR. Applied and Environmental Microbiology 75:965-969. DOI: 10.1128/AEM.02063-08. Gerdes S., Mokrousov I., Aleksic E., Allix-Béguec C., Antierens A., Augustynowicz-Kopeć

E., Ballif M., Barletta F., Beck HP., Barry CE., Bonnet M., Borroni E., Campos-Herrero I., Cirillo D., Cox H., Crowe S., Crudu V., Diel R., Drobniewski F., Fauville-Dufaux M., Gagneux S., Ghebremichael S., Hanekom M., Hoffner S., Jiao W., Kalon S., Kohl T a., Kontsevaya I., Lillebæk T., Maeda S., Nikolayevskyy V., Rasmussen M., Rastogi N., Samper S., Sanchez-Padilla E., Savic B., Shamputa IC., Shen A., Sng L-H., Stakenas P., Toit K., Varaine F., Vukovic D., Wahl C., Warren R., Supply P., Niemann S., Wirth T. 2015. 
515 Evolutionary history and global spread of the Mycobacterium tuberculosis Beijing lineage. $516 \quad$ Nature Genetics 47:242-249. DOI: 10.1038/ng.3195.

517 Milheiriço C., Oliveira DC., De Lencastre H. 2007. Update to the multiplex PCR strategy for 518 assignment of mec element types in Staphylococcus aureus. Antimicrobial Agents and Chemotherapy 51:3374-3377. DOI: 10.1128/AAC.00275-07.

Moore AM., Ahmadi S., Patel S., Gibson MK., Wang B., Ndao MI., Deych E., Shannon W., Tarr PI., Warner BB., Dantas G. 2015. Gut resistome development in healthy twin pairs in the first year of life. Microbiome 3:27. DOI: 10.1186/s40168-015-0090-9.

Penders J., Stobberingh EE., Savelkoul PHM., Wolffs PFG. 2013. The human microbiome as a reservoir of antimicrobial resistance. Frontiers in Microbiology 4. DOI: 10.3389/fmicb.2013.00087.

Qin Q-L., Li Y., Zhang Y-J., Zhou Z-M., Zhang W-X., Chen X-L., Zhang X-Y., Zhou B-C., Wang L., Zhang Y-Z. 2011. Comparative genomics reveals a deep-sea sediment-adapted life style of Pseudoalteromonas sp. SM9913. The ISME journal 5:274-284. DOI: 10.1038/ismej.2010.103.

R Developement Core Team. 2015. R: A Language and Environment for Statistical Computing. $R$ Foundation for Statistical Computing 1:409. DOI: 10.1007/978-3-540-74686-7.

Rose G., Wooldridge DJ., Anscombe C., Mee ET., Misra R V., Gharbia S. 2015. Challenges of the Unknown : Clinical Application of Microbial Metagenomics. International Journal of Genomics 2015:1-10. DOI: 10.1155/2015/292950.

Shoemaker NB., Vlamakis H., Hayes K., Salyers a a. 2001. Evidence for Extensive Resistance Gene Transfer among Bacteroides spp . and among Bacteroides and Other Genera in the 
537 Human Colon Evidence for Extensive Resistance Gene Transfer among Bacteroides spp . 538 and among Bacteroides and Other Genera in the Human C. Appl Environ Microbiol 67:561539 8. DOI: 10.1128/AEM.67.2.561.

540 Stecher B., Denzler R., Maier L., Bernet F., Sanders MJ., Pickard DJ., Barthel M., Westendorf 541 AM., Krogfelt KA., Walker AW., Ackermann M., Dobrindt U., Thomson NR., Hardt W-D.

542 2012. Gut inflammation can boost horizontal gene transfer between pathogenic and commensal Enterobacteriaceae. Proceedings of the National Academy of Sciences of the United States of America 109:1269-74. DOI: 10.1073/pnas.1113246109.

Tanaka S., Kobayashi T., Songjinda P., Tateyama A., Tsubouchi M., Kiyohara C., Shirakawa T., Sonomoto K., Nakayama J. 2009. Influence of antibiotic exposure in the early postnatal period on the development of intestinal microbiota. FEMS Immunology and Medical Microbiology 56:80-87. DOI: 10.1111/j.1574-695X.2009.00553.x.

Trobos M., Lester CH., Olsen JE., Frimodt-M??1ler N., Hammerum AM. 2009. Natural transfer of sulphonamide and ampicillin resistance between Escherichia coli residing in the human intestine. Journal of Antimicrobial Chemotherapy 63:80-86. DOI: 10.1093/jac/dkn437.

Truong DT., Franzosa EA., Tickle TL., Scholz M., Weingart G., Pasolli E., Tett A., Huttenhower

de Vries LE., Valles Y., Agerso Y., Vaishampayan PA., Garcia-Montaner A., Kuehl J V.,

556 Christensen H., Barlow M., Francino MP. 2011. The gut as reservoir of antibiotic resistance:

557 Microbial diversity of tetracycline resistance in mother and infant. PLoS ONE 6. DOI:

558 10.1371/journal.pone.0021644. 
559 Walson JL., Marshall B., Pokhrel BM., Kafle KK., Levy SB. 2001. Carriage of antibiotic-

560 resistant fecal bacteria in Nepal reflects proximity to Kathmandu. The Journal of infectious

561 diseases 184:1163-1169. DOI: 10.1086/323647.

562 von Wintersdorff CJ., Wolffs PF., Savelkoul PH., Nijsen RR., Lau S., Gerhold K., Hamelmann E.,

563 Penders J. 2016. The gut resistome is highly dynamic during the first months of life. Future

564 microbiology 11:501-510. DOI: $10.2217 / \mathrm{fmb} .15 .154$.

565 Zhang L., Kinkelaar D., Huang Y., Li Y., Li X., Wang HH. 2011. Acquired antibiotic resistance:

566 Are we born with it? Applied and Environmental Microbiology 77:7134-7141. DOI:

$567 \quad$ 10.1128/AEM.05087-11. 


\section{Figure 1 (on next page)}

Healthy premature infant gut microbiome
A) Metagenomic profiles for the eleven preterm samples and four replicates at the species level. Samples clustered by UPGMA using Bray-Curtis distances shown on left, with replicates highlighted by filled nodes. Relative abundances by rank order shown on right, with the top 8 most abundant species coloured and labelled, leaving remaining species in white. B)
Dominant species, based on $>=50 \%$ abundance, shown on $x$-axis, with overall prevalence of the species across samples shown on y-axis. Sample number reflects eleven neonates as replicates are averaged. The five labelled species are present in five or more samples with at least one in $>50 \%$ abundance. C) Principle coordinates analysis (PCOA) using Bray-Curtis distances at the species level for all fifteen samples. Separation of the three broad sample groups shown by biplot of the top five species. 

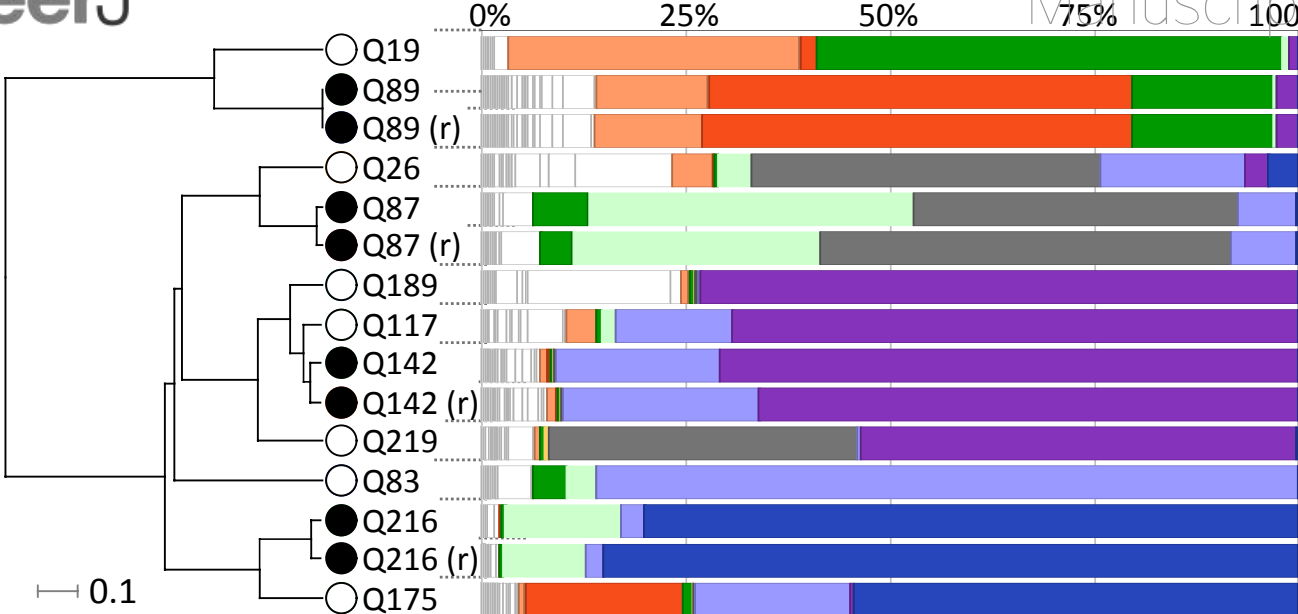

Clostridium perfringens Klebsiella pneumonia Enterococcus faecalis Bifidobacterium breve $\square$ Staphylococcus aureus Staphylococcus epidermidis Enterobacter cloacae $\longmapsto 0.1$ Q175

B

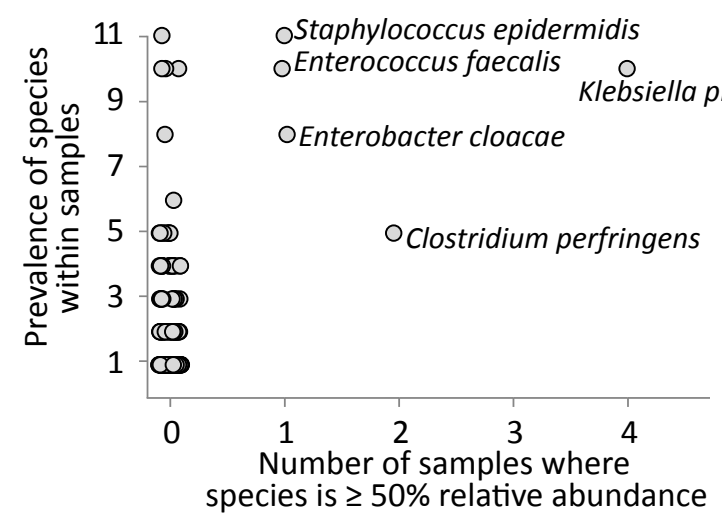
species is $\geq 50 \%$ relative abundance
C

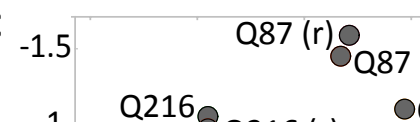

$-1$

ฉ̀- -0.5 Clostridium perfringens $\square$ Escherichia coli

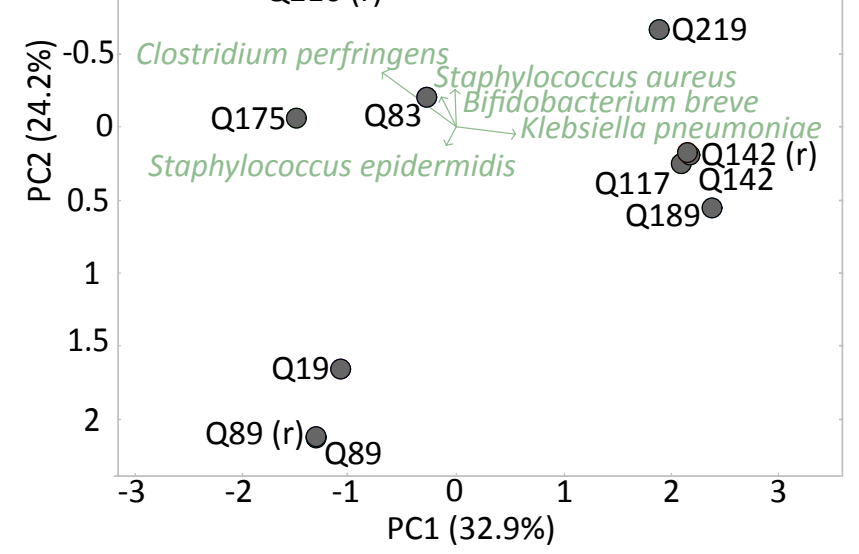


Figure 2 (on next page)

Antibiotic resistance genes detected.

Heatmap showing distribution of the 39 AMR genes detected within the eleven metagenomic samples. Genes grouped by antibiotic class: AGly (aminoglycosides), Bla (beta-lactamases), Fos (Fosfomycin), Flq (fluoroquinolones), MLS (macrolide-lincosamide- streptogramin), Phe (phenicols), Tet (tetracyclines), Tmt (trimethoprim). Colours show read depth in bp:

undetected (grey), 3-199 bp (blue), 200 - 399 bp (yellow), 400 - 1437 (red). Rows clustered by UPGMA method using Euclidean distances. 
3

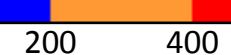

1437

AGly

Bla

Fos Flq

MLS

Phe Tet Tmt

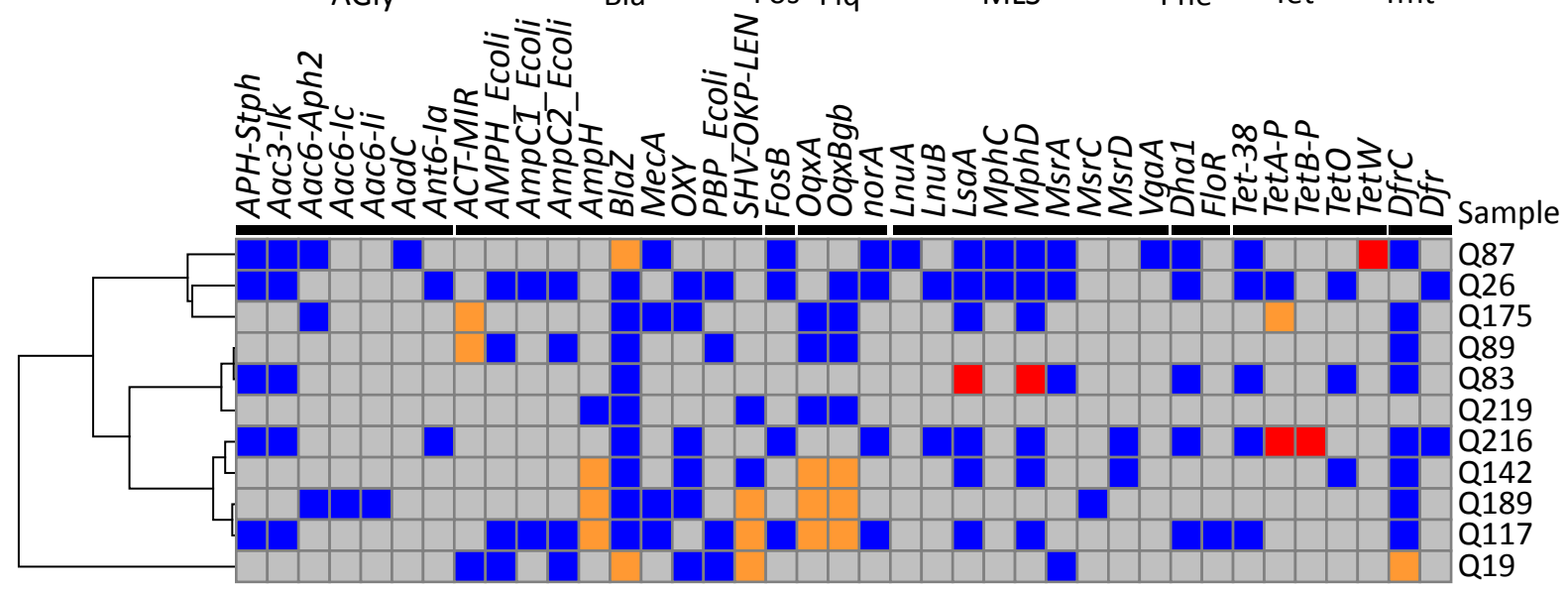




\section{Table 1 (on next page)}

Metagenomic study dataset.

Sequencing results for the eleven preterm samples sequenced and four replicates. 


\begin{tabular}{llcccccc}
\hline Sample & Instrument & $\begin{array}{c}\text { Read } \\
\text { length }\end{array}$ & Raw PE reads & $\begin{array}{c}\text { Surviving } \\
\text { PE reads* }\end{array}$ & $\begin{array}{c}\text { Surviving PE } \\
\text { reads \%* }\end{array}$ & $\begin{array}{c}\text { Mean read } \\
\text { length (bp) }\end{array}$ & $\begin{array}{c}\text { Yield } \\
\text { (Gbps) }\end{array}$ \\
\hline Q19 & NextSeq & 151 & $10,765,181$ & $9,748,620$ & 90.6 & 118.5 & 2.3 \\
Q26 & NextSeq & 151 & $10,515,261$ & $9,675,994$ & 92.0 & 125.3 & 2.4 \\
Q83 & NextSeq & 151 & $10,272,541$ & $9,434,574$ & 91.8 & 128.1 & 2.4 \\
Q87 & NextSeq & 151 & $9,771,928$ & $9,031,166$ & 92.4 & 126.3 & 2.3 \\
Q89 & NextSeq & 151 & $10,573,383$ & $9,686,447$ & 91.6 & 130.5 & 2.5 \\
Q89 (r) & NextSeq & 151 & $10,746,440$ & $9,824,221$ & 91.4 & 129.1 & 2.5 \\
Q117 & NextSeq & 151 & $9,718,743$ & $8,943,195$ & 92.0 & 124.4 & 2.2 \\
Q142 & NextSeq & 151 & $9,847,000$ & $9,059,108$ & 92.0 & 129.0 & 2.3 \\
Q175 & NextSeq & 151 & $11,442,761$ & $10,404,737$ & 90.9 & 121.1 & 2.5 \\
Q189 & NextSeq & 151 & $9,385,745$ & $8,667,469$ & 92.3 & 125.2 & 2.2 \\
Q216 & NextSeq & 151 & $11,842,425$ & $10,963,555$ & 92.6 & 125.8 & 2.8 \\
Q219 & NextSeq & 151 & $5,761,388$ & $5,295,708$ & 91.9 & 118.4 & 1.3 \\
Q87 (r) & MiSeq & 301 & $8,061,151$ & $5,710,557$ & 70.8 & 193.0 & 1.1 \\
Q142 (r) & MiSeq & 301 & $4,101,014$ & $3,234,121$ & 78.9 & 204.1 & 0.7 \\
Q216 (r) & MiSeq & 301 & $12,778,363$ & $10,848,709$ & 84.9 & 224.0 & 2.4 \\
\hline Mean & - & - & $9,705,555$ & $8,701,879$ & - & - & 2.1 \\
Total & - & - & $145,583,324$ & $130,528,181$ & 89.7 & - & - \\
\hline
\end{tabular}

2

3 * Three MiSeq replicate samples paired reads were merged during QC, therefore read number represent 4 single reads

(r) signifies replicate samples 


\section{Table 2 (on next page)}

Antibiotic classes identified.

Major antibiotic resistant classes of genes identified within the eleven samples by srst2.

Columns show antibiotic type and number of genes found within class. 
1

\begin{tabular}{lc}
\hline Antibiotic type & $\begin{array}{c}\text { Number of identified } \\
\text { genes within class }\end{array}$ \\
\hline Beta-lactamases (Bla) & 10 \\
Macrolide-lincosamide- streptogramin (MLS) & 9 \\
Aminoglycosides (AGly) & 7 \\
Tetracyclines (Tet) & 5 \\
Fluoroquinolones (Flq) & 3 \\
Phenicols (Phe) & 2 \\
Trimethoprim (Tmt) & 2 \\
Fosfomycin (Fcyn) & 1 \\
\hline
\end{tabular}
2 